\title{
SOLVABLE GROUPS ADMITTING A FIXED-POINT-FREE AUTOMORPHISM OF PRIME POWER ORDER
}

\author{
FLETCHER GROSS
}

1. Introduction. The purpose of the paper is to prove the following:

TheOREM 1. Suppose $G$ is a finite group which admits an automorphism $\sigma$ of order $p^{n}$, where $p$ is an odd prime, such that $\sigma$ fixes only the identity element of $G$.

(a) If $G$ is solvable, then $h(G) \leqq n$.

(b) If $G$ is $\pi$-solvable, then $l_{\pi}(G) \leqq[(n+1) / 2]$.

Furthermore, both these inequalities are best-possible.

Here $h(G)$, the Fitting height (also called the nilpotent length) of $G$, is as defined in [7]. $l_{\pi}(G)$, the $\pi$-length of $G$, is defined in an obvious analogy to the definition of $p$-length in [2].

Higman [3] proved Theorem 1 in the case $n=1$ (subsequently, without making any assumptions on the solvability of $G$, Thompson [6] obtained the same result). Hoffman [4] and Shult [5] proved Theorem 1 provided that either $p$ is not a Fermat prime or a Sylow 2-group of $G$ is abelian.

For $p=2$, Gorenstein and Herstein [1] obtained Theorem 1 if $n \leqq 2$, and Hoffman and Shult both obtained Theorem 1 provided that a Sylow $q$-group of $G$ is abelian for all Mersenne primes $q$ which divide the order of $G$. Shult, who considers a more general situation of which Theorem 1 is a special case, recently extended his results to include all primes, but his bound on $h(G)$ is not best-possible in the special case of Theorem 1. It also should be noted that Thompson [7] obtained a bound for $h(G)$ under a much more general hypothesis than that considered in the other papers mentioned.

Theorem 1 is a consequence of

THEOREM 2. Let $G$ be a finite group admitting a fixed-point-free automorphism $\sigma$ of order $p^{n}, p$ an odd prime, and let $H$ be a normal Hall subgroup of $G$ such that $H$ contains its centralizer in $G$. Then the automorphism of $G / H$ induced by $\sigma^{p^{n-1}}$ is the identity automorphism.

Here again, the papers of Hoffman and Shult imply Theorem 2 if either $p$ is not a Fermat prime or a Sylow 2-group of $G$ is abelian. Thus what is new about the present paper is that no condition is imposed upon the Sylow 2-groups of $G$ if $p$ is a Fermat prime.

Received by the editors February 15, 1966. 
The restriction to odd primes is essential since Theorem 2 is false for $p=2, n>2$. To see this let $q=2^{m}-1$ be some Mersenne prime and let $M$ be the nonabelian group of exponent $q$ and order $q^{3} . M$ admits a fixed-point-free automorphism $\sigma$ of order $2^{m+1}$. Let $K$ be the semidirect product of $M$ and the group generated by $\sigma^{2}$, and choose $F$ to be any finite field such that (1) the characteristic of $F$ is not 2 or $q$, and (2) $F$ is a splitting field for $K$. There is a faithful irreducible representation $\rho$ of $K$ over $F$ such that $\rho\left(\sigma^{2}\right)$ has no nonzero fixed vectors. Now for $x \in M$, define $\rho^{*}(x)$ by $\rho^{*}(x)=\rho(x) \oplus \rho\left(x^{\sigma}\right)$. Then choose $\rho^{*}(\sigma)$ to be

$$
\left(\begin{array}{rr}
0 & \rho\left(\sigma^{2}\right) \\
I & 0
\end{array}\right)
$$

$\rho^{*}(\sigma)$ is of order $2^{m+1}$ and $\rho^{*}(\sigma)^{-1} \rho^{*}(x) \rho^{*}(\sigma)=\rho^{*}\left(x^{\sigma}\right)$. Thus $\rho^{*}$ is a faithful representation of the semidirect product of $M$ and $\langle\sigma\rangle$, the group generated by $\sigma$. If $H$ is the space on which $\rho^{*}(M\langle\sigma\rangle)$ operates, then $\rho^{*}(\sigma)$ induces a fixed-point-free automorphism of the semidirect product $H M$. This automorphism is of order $2^{m+1}$ on both $H M$ and $H M / H$.

2. Proofs. First we need some elementary number theoretic results which we state without proof.

(2.1) Lemma. Let $p=2^{s}+1$ be an odd Fermat prime. Then $p^{k}$ divides $\left(2^{n}-1\right)$ if and only if $2 s p^{k-1}$ divides $n$.

(2.2) Lemma. Suppose $p=2^{*}+1$ is an odd Fermat prime and $p^{a}$ $=2^{b}+1$ for some positive integers $a, b$. Then $a=1, b=s$ unless $p=3$, in which case $a=2, b=3$ is also possible.

We now proceed to prove Theorem 2 by induction on the order of $G$. $H$ is a characteristic subgroup of $G$ so $H$ certainly admits $\sigma$. By induction, if $G_{1}$ is a proper subgroup of $G$ such that $G_{1}$ admits $\sigma$ and $G_{1} \geqq H$, then $\sigma^{x^{n-1}}$ must be the identity on $G_{1} / H$. According to [2, Theorem C], this implies that

(1) $G / H$ is a $q$-group for some prime $q$.

(2) Either $\phi(G / H)=1$ or $(G / H)^{\prime}=\phi(G / H)=Z(G / H)$.

(3) $\langle\sigma\rangle$ is faithfully and irreducibly represented by the automorphisms induced on $(G / H) / \phi(G / H)$.

(Here $\phi(G)$ and $Z(G)$ denote the Frattini subgroup and center, respectively, of $G$.) Now there must be a Sylow $q$-group $M$ of $G$ such that $M$ admits $\sigma$. Clearly $G=H M$ and $M \cong G / H$. Thus we must show that $\sigma^{p^{n-1}}$ fixes $M$ elementwise. For convenience we set $\sigma^{p^{n-1}}=\sigma^{\prime}$.

Now suppose $x$ is an element of $M$ not fixed by $\sigma^{\prime}$. Let $y=\left(x, \sigma^{\prime}\right)$ 
$=x^{-1} x^{\sigma^{\prime}} \neq 1$. Now since $H$ contains its centralizer in $G$, it follows that there is a Sylow $r$-group $K$ of $H$ such that $M$ normalizes $K, K$ admits $\sigma$, and $(y, K) \neq 1$. Now let $N$ be the centralizer of $K$ in $M$, and consider the group $K M / N$. This group satisfies the hypothesis of Theorem 2, and so, if $H \neq K$, we must have $\sigma^{\prime}$ is the identity on $M / N$. But $N$ is a proper subgroup of $M$ (since $y \notin N$ ) so that $\sigma^{\prime}$ must fix $N$ elementwise. Since $p$ cannot divide the order of $M$, this would imply that $\sigma^{\prime}$ is the identity on $M$.

Thus we assume that $H$ is an $r$-group for some prime $r$. Now $G / \phi(H)$ satisfies the hypothesis of the theorem, so by induction we may assume that $\phi(H)=1$. From now on we consider $H$ as a vector space over a field $F$ of characteristic $r$ and we consider $M\langle\sigma\rangle$, the semidirect product of $M$ and $\langle\sigma\rangle$, as a linear group operating on $H$. Since $\sigma$ is fixed-point-free on $G, \sigma$, as a linear transformation, cannot have 1 as an eigenvalue. Now extending the field $F$ does not change the structure of $\langle\sigma\rangle M$ nor the eigenvalues of $\sigma$. Accordingly we consider $\langle\sigma\rangle M$ as a linear group over a field $F$ of characteristic $r$, and we assume that $F$ is a splitting field for $\langle\sigma\rangle M$.

Now let $V$ be an irreducible $F-\langle\sigma\rangle M$ submodule such that $\left(M, \sigma^{p^{n-1}}\right)$ is not the identity on $V$. Next decompose $V$ into the sum $V=V_{1} \oplus V_{2} \oplus \cdots$ of minimal characteristic $F-M$ submodules $V_{\boldsymbol{i}}$. Since $V$ is irreducible, $\sigma$ must permute the $V_{i}$ transitively. Let $\tau=\sigma^{p^{m}}$ be the first power of $\sigma$ which fixes all the $V_{i}$, and number the $V_{i}$ so that $V_{i} \sigma=V_{i+1}\left(\bmod p^{m}\right)$. Next let $N$ be the restriction of $M$ to $V, K_{i}$ the kernel of the representation of $N$ afforded by the module $V_{i}$, and $Q_{i}=N / K_{i}$. Since $Z\left(Q_{i}\right)$ is represented by a scalar matrix on $V_{i}, \tau$ must fix $Z\left(Q_{i}\right)$ elementwise. Now $m=0$ would imply that $\tau=\sigma$, $V=V_{1}$, and $K_{1}=1$. Since $\sigma$ must induce a fixed-point-free automorphism of $Z(N)$, this implies that $m>0$.

Now the argument in [5, pp. 704-708] shows that 1 must be an eigenvalue of $\sigma$ unless $p^{n-m}=q^{d}+1, Q_{i}$ is of order $q^{2 d+1}$, and $Q_{i}$ is an extra-special $q$-group. We now proceed to finish the proof of Theorem 2 by showing that under the conditions just stated, $\sigma$ cannot be fixedpoint-free on $N^{\prime}$.

First $p^{n-m}=q^{d}+1$ implies that $q=2$ (since $p$ is odd) and $p$ is a Fermat prime $=2^{s}+1$. Thus either $d=s, n-m=1$ or, if $p=3$, we could have $d=3, n-m=2$. In any event $d$ is the smallest positive integer such that $\left(2^{2 d}-1\right)$ is divisible by $p^{n-m}$. Now $\sigma^{p^{n-1}}$ is not the identity on any $V_{i}$ since $\left(M, \sigma^{p^{n-1}}\right)$ is not the identity on $V$. For the same reason $N / N^{\prime}$ is a faithful $G F(q)-\langle\sigma\rangle$ module and $Q_{i} / Q_{i}^{\prime}$ is a faithful $G F(q)-\langle\tau\rangle$ module. But since $M / \phi(M)$ is an irreducible module for $\langle\sigma\rangle$ and since $Q_{i} / Q_{i}^{\prime}$ is of order $2^{2 d}$, it follows that $N / N^{\prime}$ 
and $Q_{i} / Q_{i}^{\prime}$ are irreducible modules for $\langle\sigma\rangle$ and $\langle\tau\rangle$, respectively. From (2.1) it follows that the smallest in teger $k$ such that $p^{n}$ divides $\left(2^{k}-1\right)$ is $k=2 s p^{n-1}=2 d p^{m}$. Thus $N / N^{\prime}$ is of order $2^{2 d p^{m}}$. Now $Q_{i}=N / K_{i}$ and so $Q_{i} / Q_{i}^{\prime}$ is operator isomorphic as a $\langle\tau\rangle$-module to $N /\left(K_{i} N^{\prime}\right)$.

(2.3) Lemma. (1) For all $i, k$ such that $1 \leqq i \leqq p^{m}, 1 \leqq k \leqq p^{m}$,

$$
\left(\bigcap_{j=i}^{i+k-1} K_{j} N^{\prime}\right) / N^{\prime}
$$

is of order $2^{2 d\left(p^{m}-k\right)}$.

(2) For all $i, k$ such that $1 \leqq i \leqq p^{m}, 1 \leqq k<p^{m}$,

$$
\left(\bigcap_{j=i}^{i+k-1} K_{j} N^{\prime}\right)\left(K_{i+k} N^{\prime}\right)=N .
$$

Proof. Throughout, the indices $j$ on the subgroups $K_{j}$ are to be taken modulo $p^{m}$. Now if $k=1$, then

$$
\left|K_{i} N^{\prime} / N^{\prime}\right|=\left|N / N^{\prime}\right| /\left|N / K_{i} N^{\prime}\right|=2^{2 d\left(p^{m}-1\right)} .
$$

Now assume the first assertion of the lemma is true for a given $k<p^{m}$. Now

$$
\left(\bigcap_{j=i}^{i+k-1} K_{j} N^{\prime}\right) K_{i+k} N^{\prime} / K_{i+k} N^{\prime}
$$

is a $\langle\tau\rangle$-submodule of $N / K_{i+k} N^{\prime}$. Since $N / K_{i+k} N^{\prime}$ is an irreducible $\langle\tau\rangle$-module, we conclude that either the second part of the lemma holds or

$$
\bigcap_{j=i}^{i+k-1} K_{j} N^{\prime} \leqq K_{i+k} N^{\prime} .
$$

In the latter case we certainly have

$$
\bigcap_{j=i}^{i+k-1} K_{j} N^{\prime} \leqq \bigcap_{j=i+1}^{i+k} K_{j} N^{\prime}=\left(\bigcap_{j=i}^{i+k-1} K_{j} N^{\prime}\right)^{\prime}
$$

Since

$$
N>\left(\bigcap_{j=i}^{i+k-1} K_{j} N^{\prime}\right)>N^{\prime}
$$

from (1), this implies that

$$
\left(\bigcap_{j=i}^{i+k-1} K_{j} N^{\prime}\right) / N^{\prime}
$$


is a nontrivial proper $\langle\sigma\rangle$-submodule of the irreducible $\langle\sigma\rangle$-module $N / N^{\prime}$. This contradiction establishes the second part of the lemma for the given value of $k$.

But then

$$
N / K_{i+k} N^{\prime} \cong\left(\bigcap_{j=i}^{i+k-1} K_{j} N^{\prime}\right) /\left(\bigcap_{j=i}^{i+k} K_{j} N^{\prime}\right)
$$

But since

$$
\left|\left(\bigcap_{j=i}^{i+k-1} K_{j} N^{\prime}\right) / N^{\prime}\right|=2^{2 d\left(p^{m}-k\right)} \text { and }\left|N / K_{i+k} N^{\prime}\right|=2^{2 d}
$$

this implies that

$$
\left|\left(\bigcap_{j=i}^{i+k} K_{j} N^{\prime}\right) / N^{\prime}\right|=2^{2 d\left(p^{m}-k-1\right)}
$$

Thus part (1) of the lemma is proved for $k+1$. Then, by induction, the lemma is proved.

Now let $L_{i}=\bigcap_{j \neq i} K_{j} N^{\prime}$ for all $i, 1 \leqq i \leqq p^{m}$. From the lemma, $L_{i} K_{i}$ $=L_{i} K_{i} N^{\prime}=N$ for all $i$. Also since $L_{i}^{\sigma}=L_{i+1}\left(\bmod p^{m}\right), L_{1} L_{2} \cdots L_{p^{m}} / N^{\prime}$ is a nontrivial $\langle\sigma\rangle$-module. Thus $L_{1} L_{2} \cdots L_{p^{m}}=N$. Our goal now is to show that $N^{\prime}$ is the direct product

$$
L_{1}^{\prime} \times L_{2}^{\prime} \times \cdots \times L_{p^{m}}^{\prime} .
$$

To do this, we first need

(2.4) Lemma. $\left(L_{i}, L_{k}\right)=1$ if $i \neq k$.

Proof. Suppose $(x, y) \neq 1$ for $x \in L_{i}, y \in L_{k}$. Choose $t$ such that $(x, y)$ is not the identity on $V_{t}$. Now at least one of $L_{i}$ and $L_{k}$ is contained in $K_{t} N^{\prime}$. Without loss of generality assume that $L_{i} \leqq K_{t} N^{\prime}$. Therefore $x=g h$ where $g \in K_{t}, h \in N^{\prime}$. Now $N^{\prime} \leqq Z(N)$. Therefore $(g h, y)=(g, y)$. But $g$ is the identity on $V_{t}$ which implies that $(g, y)$ is also the identity on $V_{t}$. This proves the lemma.

As an immediate consequence of the lemma we have $N^{\prime}=L_{1}^{\prime} L_{2}^{\prime}$ ... $L_{p^{m}}^{\prime}$. Now as in the proof just given, $L_{i} \leqq K_{t} N^{\prime}$ implies that $\left(L_{i}\right.$, $N)$ is the identity on $V_{t}$. Since $N$ is faithfully represented on $V$, this implies that $L_{i}^{\prime}$ is faithfully represented on $V_{i}$. Thus

$$
\left|L_{i}^{\prime}\right|=\left|Q_{i}^{\prime}\right|=2 \quad \text { for all } i .
$$

Now suppose $L_{i}^{\prime} \cap \Pi_{j \neq i} L_{j}^{\prime} \neq 1$. Then we would have $\Pi_{j \neq i} L_{j}^{\prime}$ not the identity on $V_{i}$. But $j \neq i$ implies that $L_{j}^{\prime} \leqq\left(L_{j}, N\right)$ is the identity on $V_{i}$. Thus $L_{i}^{\prime} \cap \Pi_{j \neq i} L_{j}^{\prime}=1$ for all $i$. This implies that 


$$
N^{\prime}=L_{1}^{\prime} \times L_{2}^{\prime} \times \cdots \times L_{p^{m}}^{\prime}
$$

and thus $\left|N^{\prime}\right|=2^{p^{m}}$. Since $\left|N^{\prime}\right| \not \equiv 1(\bmod p), N^{\prime}$ cannot have a fixedpoint-free automorphism whose order is a power of $p$. This concludes the proof of Theorem 2 .

The proof of Theorem 1 now follows by induction on the order of $G$. First suppose $G$ has two distinct minimal $\sigma$-admissible normal subgroups $H_{1}, H_{2}$. Then $G$ is isomorphic to a subgroup of the direct product of $G / H_{1}$ and $G / H_{2}$ and both $G / H_{1}$ and $G / H_{2}$ satisfy the theorem. It then follows that $G$ would satisfy the theorem.

Thus, for part (a) of the theorem, we may assume that the Fitting group $F_{1}(G)$ is a $q$-group for some prime $q$. Then $O_{q q^{\prime}}(G)$ satisfies the conditions of Theorem 2. Therefore $\sigma^{p^{n-1}}$ is the identity on $O_{q q^{\prime}}(G) / F_{1}(G)$. By $[4$, Lemma 4$]$, this implies that $\sigma^{p^{n-1}}$ is the identity on $G / F_{1}(G)$. Then, by induction, we have

$$
h(G)=1+h\left(G / F_{1}(G)\right) \leqq 1+(n-1)=n .
$$

For part (b) of Theorem 1, we may assume that $O_{\pi^{\prime}}(G)=1$. Then by one application of Theorem $2, \sigma^{p^{n-1}}$ is the identity on $O_{\pi \pi^{\prime}}(G) / O_{\pi}(G)$, and by a second application, $\sigma^{p^{n-2}}$ is the identity on $O_{\pi \pi^{\prime} \pi}(G) / O_{\pi \pi^{\prime}}(G)$. Thus, again using $[4$, Lemma 4$], \sigma^{p^{n-2}}$ is the identity on $G / O_{\pi \pi^{\prime}}(G)$. Induction now implies that

$$
l_{\pi}(G)=1+l_{\pi}\left(G / O_{\pi \pi^{\prime}}(G)\right) \leqq 1+[(n-1) / 2]=[(n+1) / 2] .
$$

All that remains now is to show that the inequalities in Theorem 1 are best-possible. For part (a), this follows from examples constructed by Shult [5, Theorem 5]. For part (b), however, Shult's construction has to be modified somewhat. Working by induction, Shult assumes that $G_{k}$ is a solvable group of Fitting height $k$ which admits a fixedpoint-free automorphism of order $p^{k}$. Then if $q_{k}$ is any prime such that $q_{k} \equiv 1(\bmod p)$ and $q_{k}$ does not divide the order of $G$, Shult proceeds to construct a new group $G_{k+1}$ such that $F_{1}\left(G_{k+1}\right)$ is a $q$-group, $G_{k+1} / F_{1}\left(G_{k+1}\right)$ is isomorphic to $G_{k}, h\left(G_{k+1}\right)=k+1$, and $G_{k+1}$ admits a fixed-point-free automorphism of order $p^{k+1}$. A close look at Shult's procedure reveals that it is only necessary that $q_{k}$ does not divide the order of $F_{1}(G)$. Thus if $q, r$ are distinct primes such that $q \equiv r \equiv 1$ $(\bmod p)$, Shult's procedure can be used to construct groups $G_{k}$ with the following properties.

(1) $G_{k}$ is a $q, r$-group.

(2) $F_{1}\left(G_{k}\right)$ is either a $q$ - or an $r$-group.

(3) $G_{k}$ admits a fixed-point-free automorphism of order $p^{k}$.

(4) $h\left(G_{k}\right)=k$. 
It now follows that $l_{q}\left(G_{k}\right)$ and/or $l_{r}\left(G_{k}\right)$ is equal to $[(k+1) / 2]$. Thus the inequality in part (b) is best-possible.

\section{REFERENCES}

1. D. Gorenstein and I. Herstein, Finite groups admitting a fixed-point-free automorphism of order 4, Amer. J. Math. 83 (1961), 71-78.

2. P. Hall and G. Higman, On the p-length of p-soluble groups and reduction theorems for Burnside's problem, Proc. London Math. Soc. (3) 6 (1956), 1-42.

3. G. Higman, Groups and rings having automorphisms without non-trivial fixed elements, J. London Math. Soc. 32 (1958), 321-334.

4. F. Hoffman, Nilpotent height of finite groups admitting fixed-point-free automorphisms, Math. Z. 85 (1964), 260-267.

5. E. Shult, On groups admitting fixed point free abelian groups, Illinois J. Math. 9 (1965), 701-720.

6. J. Thompson, Finite groups with fixed-point-free automorphisms of prime order, Proc. Nat. Acad Sci. U.S.A. 45 (1959), 578-581.

7. —-, Automorphisms of solvable groups, J. Algebra 1 (1964), 259-267.

University of Alberta, Edmonton 